\title{
(GIGA)bYte
}

\section{Chromosome-level genome assembly of a benthic associated Syngnathiformes species: the common dragonet, Callionymus lyra}

Sven Winter ${ }^{1,2, *}$, Stefan Prost ${ }^{3,4}$, Jordi de Raad ${ }^{1,2,3}$, Raphael T. F. Coimbra ${ }^{1,2}$, Magnus Wolf ${ }^{1,2}$, Marcel Nebenführ ${ }^{1}$, Annika Held ${ }^{1}$, Melina Kurzawe ${ }^{1}$, Ramona Papapostolou ${ }^{1}$, Jade Tessien ${ }^{1}$, Julian Bludau ${ }^{1}$, Andreas Kelch ${ }^{1}$, Sarah Gronefeld ${ }^{1}$, Yannis Schöneberg ${ }^{1}$, Christian Zeitz ${ }^{1}$, Konstantin Zapf ${ }^{1}$, David Prochotta ${ }^{1}$, Maximilian Murphy ${ }^{1}$, Monica M. Sheffer ${ }^{5}$, Moritz Sonnewald ${ }^{6}$, Maria A. Nilsson ${ }^{2,3}$ and Axel Janke ${ }^{1,2,3}$

\section{Institute for Ecology, Evolution and Diversity, Goethe University, Frankfurt am Main, Germany Senckenberg Biodiversity and Climate Research Centre, Frankfurt am Main, Germany LOEWE-Centre for Translational Biodiversity Genomics, Frankfurt am Main, Germany South African National Biodiversity Institute, National Zoological Garden, Pretoria, South Africa Zoological Institute and Museum, University of Greifswald, Greifswald, Germany \\ 6 Senckenberg Research Institute, Department of Marine Zoology, Section Ichthyology, Frankfurt am Main, Germany}

\section{ABSTRACT}

Background: The common dragonet, Callionymus lyra, is one of three Callionymus species inhabiting the North Sea. All three species show strong sexual dimorphism. The males show strong morphological differentiation, e.g., species-specific colouration and size relations, while the females of different species have few distinguishing characters. Callionymus belongs to the 'benthic associated clade' of the order Syngnathiformes. The 'benthic associated clade' so far is not represented by genome data and serves as an important outgroup to understand the morphological transformation in 'long-snouted' syngnatiformes such as seahorses and pipefishes. Findings: Here, we present the chromosome-level genome assembly of $C$. lyra. We applied Oxford Nanopore Technologies' long-read sequencing, short-read DNBseq, and proximity-ligation-based scaffolding to generate a high-quality genome assembly. The resulting assembly has a contig N50 of $2.2 \mathrm{Mbp}$ and a scaffold N50 of $26.7 \mathrm{Mbp}$. The total assembly length is $568.7 \mathrm{Mbp}$, of which over $538 \mathrm{Mbp}$ were scaffolded into 19 chromosome-length scaffolds. The identification of $94.5 \%$ complete BUSCO genes indicates high assembly completeness. Additionally, we sequenced and assembled a multi-tissue transcriptome with a total length of $255.5 \mathrm{Mbp}$ that was used to aid the annotation of the genome assembly. The annotation resulted in 19,849 annotated transcripts and identified a repeat content of $27.7 \%$.

Conclusions: The chromosome-level assembly of $C$. lyra provides a high-quality reference genome for future population genomic, phylogenomic, and phylogeographic analyses. distributed under the terms of the Creative Commons Attribution License (https://creativecommons. org/licenses/by/4.0/), which permits unrestricted reuse, distribution, and reproduction in any medium, provided the original work is properly cited.

Gigabyte, 2020, 1-10 


\section{DATA DESCRIPTION BACKGROUND INFORMATION}

Until recently, the family Callionymidae was placed into the order Perciformes, which is often considered a 'polyphyletic taxonomic wastebasket for families not placed in other orders' [1]. However, recent phylogenetic analyses suggest a placement of Callionymidae within the order Syngnathiformes, which currently contains ten families with highly derived morphological characters such as the pipefish and seahorses [1]. Syngnathiformes has recently been divided into two clades, a 'long-snouted clade' and a 'benthic associated clade,' each comprising five families [2]. The 'long-snouted clade' (Syngnathidae, Solenostomidae, Aulostomidae, Centriscidae, and Fistulariidae) is currently represented by genomes from the Gulf Pipefish (Syngnathus scovelli) and the Tiger Tail Seahorse (Hippocampus comes) [3, 4] and additional draft assemblies of pipefish [5]. A genome of the 'benthic associated clade' (Callionymidae, Draconettidae, Dactylopteridae, Mullidae, and Pegasidae) has not been sequenced and analysed yet. Callionymidae comprises 196 species [6], of which the common dragonet, Callionymus lyra (Linnaeus, 1758) (Figure 1), is one of three Callionymus species inhabiting the North Sea [7]. All three species also occur in the East Atlantic, and the Mediterranean Sea [6]. They represent essential prey fish for commercially important fish species such as the cod (Gadus morhua) [8]. The males of the North Sea dragonet species (C. lyra, C. maculatus, C. reticulatus) show strong morphological differentiation in the form of species-specific colouration and size relations. The much less conspicuous females can be distinguished morphologically, with rather high inaccuracy, by the presence or absence of their preopercular, basal spine and by various percentual length ratios. The great resemblance among the different species' females, together with the fact that all three species can be found in sympatry, suggests there is the possibility of hybridization among them.

Here, we present the chromosome-level genome of the common dragonet, representing the first genome of the 'benthic associated' Syngnathiformes clade as a reference for future population genomic, phylogenomic, and comparative genomic analyses. The chromosome-level genome assembly was generated as part of a six-week university master's course. For a detailed description and outline of the course, see Prost et al. [9].

\section{SAMPLING, DNA EXTRACTION, AND SEQUENCING}

We sampled two Callionymus lyra (NCBI: txid34785; Fishbase ID:23) individuals (one of each sex) during a yearly monitoring expedition to the Dogger Bank in the North Sea (Female: $54^{\circ} 59.189^{\prime} \mathrm{N} 1^{\circ} 37.586^{\prime} \mathrm{E}$; Male: $54^{\circ} 48.271^{\prime} \mathrm{N} 1^{\circ} 25.077^{\prime} \mathrm{E}$ ) with the permission of the Maritime Policy Unit of the UK Foreign and Commonwealth Office in 2019. The samples were initially frozen at $-20^{\circ} \mathrm{C}$ on the ship and later stored at $-80^{\circ} \mathrm{C}$ until further processing. The study was conducted in compliance with the 'Nagoya Protocol on Access to Genetic Resources and the Fair and Equitable Sharing of Benefits Arising from Their Utilization'.

We extracted high molecular weight genomic DNA (hmwDNA) from muscle tissue of the female individual following the protocol by Mayjonade et al. [10]. Quantity and quality of the DNA was evaluated using the Genomic DNA ScreenTape on the Agilent 2200 TapeStation system (Agilent Technologies). Library preparation for long-read sequencing followed the associated protocols for Oxford Nanopore Technologies (ONT, Oxford, UK) Rapid Sequencing Kit (SQK-RAD004). A total of seven sequencing runs were performed using individual flow cells (FLO-MIN106 v.9.41) on a ONT MinION v.Mk1B. 


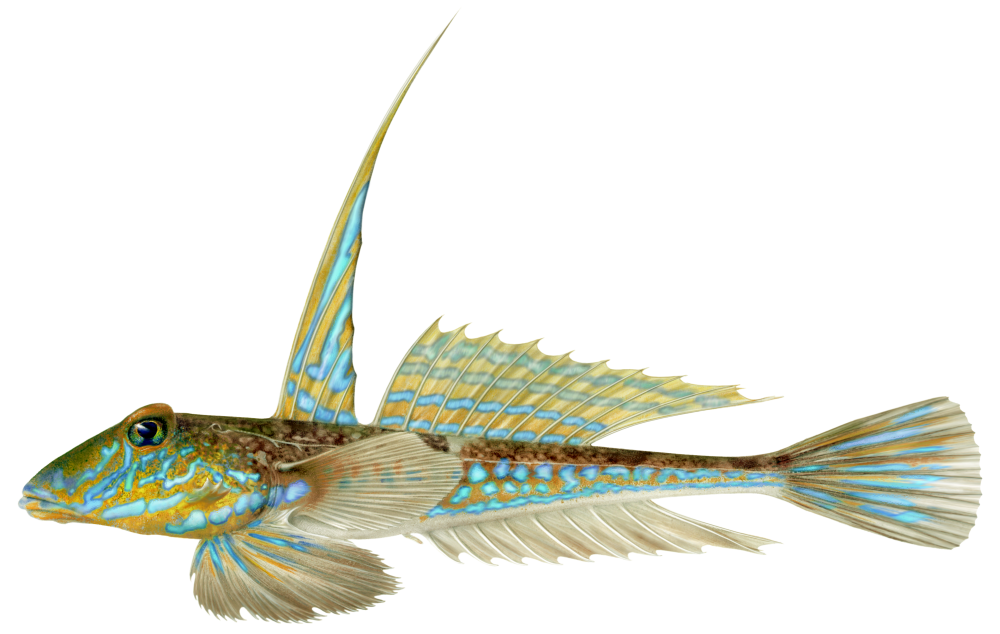

Figure 1. Male Callionymus lyra. Artwork by Karl Jilg/ArtDatabanken.

Additionally, we sent tissue samples to BGI Genomics (Shenzhen, China) to generate additional sequencing data. A 100 bp paired-end short-read genomic DNA sequencing library was prepared from the muscle tissue of the female individual. This library was later used for genome assembly polishing. Moreover, a $100 \mathrm{bp}$ paired-end RNAseq library was prepared for pooled RNA isolates derived from kidney, liver, gill, gonad, and brain tissues of the male individual. Both libraries were sequenced on BGI's DNBseq platform (BGISEQ-500/DNBSEQ-G50 sequencing) [11]. We received a total of 159,925,221 read pairs ( $32 \mathrm{Gbp}$ ) of pre-filtered genomic DNA sequencing data and 61,496,990 read-pairs ( $\sim 12.3 \mathrm{Gbp})$ of pre-filtered RNAseq data.

Furthermore, we prepared a Hi-C library using the Dovetail ${ }^{\mathrm{TM}}$ Hi-C Kit (Dovetail Genomics, Santa Cruz, California, USA) from muscle tissue of the female and sent the library to Novogene Co., Ltd. (Beijing, China) for sequencing on an Illumina NovaSeq 6000. Sequencing yielded a total of 104,668,356 pre-filtered 150 bp paired-end read pairs or 31.4 Gbp of sequencing data. This data was used for proximity-ligation scaffolding of the assembly.

\section{GENOME SIZE ESTIMATION}

We estimated the genome size for $C$. lyra using both k-mer frequencies and flow cytometry. The k-mer frequency for $K=21$ was calculated from the short-read DNBseq data and summarized as histograms with jellyfish v.2.2.10 (RRID:SCR_005491) [12]. Plotting the histograms and calculating the genome size and heterozygosity with GenomeScope v.1.0 (RRID:SCR_017014) [13] resulted in a genome size estimate of approximately $562 \mathrm{Mbp}$. For the genome size estimation using flow cytometry, frozen muscle tissue was finely chopped with a razor blade in $200 \mu \mathrm{l}$ LeukoSure Lyse Reagent (Beckman Coulter Inc., Fullerton, CA, USA). Large debris was removed by filtering through a $40 \mu \mathrm{m}$ Nylon cell strainer and an RNAse treatment was performed with a final concentration of $0.3 \mathrm{mg} / \mathrm{ml}$. Simultaneously, we stained the DNA in the nuclei with propidium iodide (PI) at a final concentration of $0.025 \mathrm{mg} / \mathrm{ml}$ and incubated the solution for $30 \mathrm{~min}$ at room temperature, protected from light exposure. Fluorescence intensities of the nuclei were recorded on the CytoFLEX Flow Cytometer (Beckman Coulter Inc., Fullerton, CA, USA). The domestic cricket (Acheta 


\begin{tabular}{|c|c|c|c|c|c|c|c|c|}
\hline & Run 1 & Run 2 & Run 3 & Run 4 & Run 5 & Run 6 & Run 7 & Total \\
\hline Mean read length & 1,153 & 1,528 & 2,562 & 2,542 & 1,913 & 1,334 & 1,211 & 1,904 \\
\hline Mean read quality: & 8.7 & 9.3 & 10.1 & 10.6 & 10.6 & 9.4 & 9.9 & 10.2 \\
\hline Number of reads: & 49,659 & 114,845 & $2,465,768$ & $2,360,176$ & $6,506,852$ & $2,149,172$ & $2,714,852$ & $16,361,324$ \\
\hline Read length N50: & 3,425 & 3,628 & 5,469 & 5,257 & 3,485 & 2,880 & 2,179 & 3,931 \\
\hline Total bases: & $57,260,945$ & $175,534,110$ & $6,316,560,588$ & $5,998,757,646$ & $12,447,462,241$ & $2,865,915,086$ & $3,287,474,510$ & $31,148,965,126$ \\
\hline
\end{tabular}

domesticus, C-value: $2.0 \mathrm{pg}$ ) was used as a reference to determine the genome size of $C$. lyra. For a more precise estimate we analysed five independent technical replicates resulting in an average C-value of $0.66 \mathrm{pg}$, which corresponds to a haploid genome size of approximately $645 \mathrm{Mbp}$.

\section{GENOME ASSEMBLY AND POLISHING}

Nanopore raw signal data (fast5) of the seven sequencing runs were base-called with Guppy v.3.2.4 (ONT) using the high accuracy setting. All individual sequencing runs were examined and compared with NanoComp v.1.0.0 [15] (Figure in GigaDB [14], Table 1).

The final dataset, after concatenation of all read-files, was further examined with NanoPlot v.1.0.0 (Table 1) [15]. Concatenation of all read-files resulted in a total dataset of $31 \mathrm{Gbp}$ or approximately 55 -fold coverage as the basis for the genome assembly.

We assembled the genome of $C$. lyra with wtdbg2 v.2.2 (RRID:SCR_017225) [16] using the default parameters for ONT reads. The resulting assembly was subjected to a three-step polishing approach. First, a single iteration of racon v.1.4.3 (RRID:SCR_017642) [17] corrected for errors typical of the MinION platform: homopolymers and repeat errors. Next, we used one iteration of medaka v.0.11.5 [18] on the racon-polished assembly. According to the developers medaka is most effective after a polishing run with racon. Following polishing with the long-read data, we used three iterations of pilon v.1.23 (RRID:SCR_014731) [19] to correct for random errors and single-base errors with the high-quality short-read data.

\section{ASSEMBLY QC AND SCAFFOLDING}

We calculated assembly continuity statistics using QUAST v.5.0.2 (RRID:SCR_001228) [20] and performed a gene set completeness analysis using BUSCO v.4.0.6

(RRID:SCR_015008) [21] with the provided database for Actinopterygii orthologous genes (actinopterygii_odb10). The final polished assembly had 1,782 contigs and a total length of $569 \mathrm{Mbp}$, which is marginally larger than the k-mer based estimate of $562 \mathrm{Mbp}$ and $84 \mathrm{Mbp}$ shorter than the flow cytometry estimate. This is expected, because very repetitive regions are usually missing or collapsed in a genome assembly, which could explain the shorter assembly length compared to the flow cytometry size estimate. The assembly shows a high continuity with long contigs of up to $10.7 \mathrm{Mbp}$ and a contig N50 of >2.2 Mbp (Table 1). The genome assembly completeness analysis identified $95.0 \%$ complete BUSCO genes ( $93.6 \%$ complete, single copy) and only $4.4 \%$ missing BUSCOs, which suggests that the assembly contains most of the coding regions of the genome (Figure 2, Table 2).

To achieve chromosome-length scaffolds, we used the long-read based assembly and the generated Hi-C data as input for the HiRise scaffolding pipeline [22] as part of the Dovetail Genomics' scaffolding service. HiRise made 538 joins and 10 breaks resulting in a scaffolded assembly with a total of 1,254 scaffolds and a scaffold N50 of $26.7 \mathrm{Mbp}$. Over $94.5 \%$ (538 


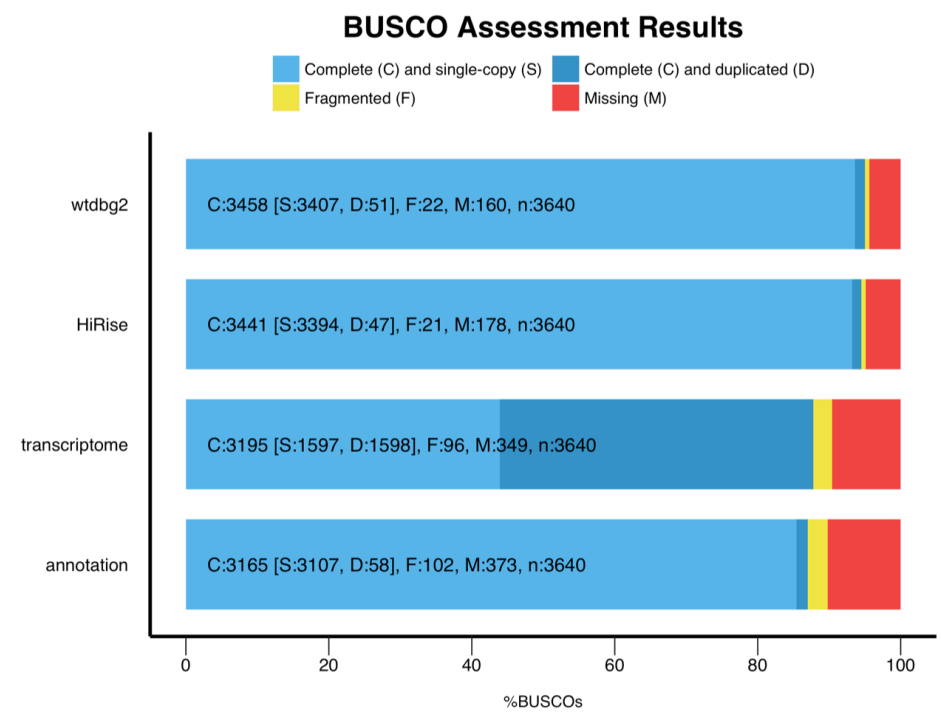

Figure 2. Gene completeness analysis of the long-read based contig assembly (wtdbg2), the Hi-C scaffolded assembly (HiRise), the transcriptome of Callionymus lyra, and the annotation. The high percentage of duplicated BUSCOs in the transcriptome is attributed to protein isoforms.

\begin{tabular}{|c|c|c|c|c|}
\hline & wtdbg2 & HiRise $^{*}$ & Transcriptome & Annotation \\
\hline Complete BUSCOs & $3458(95.0 \%)$ & $3441(94.5 \%)$ & $3195(87.8 \%)$ & $3165(87.0 \%)$ \\
\hline Complete and single-copy BUSCOs & $3407(93.6 \%)$ & $3394(93.2 \%)$ & $1597(43.9 \%)$ & $3107(85.4 \%)$ \\
\hline Complete and duplicated BUSCOs & $51(1.4 \%)$ & $47(1.3 \%)$ & $1598(43.9 \%)$ & $58(1.6 \%)$ \\
\hline Fragmented BUSCOs & $22(0.6 \%)$ & $21(0.6 \%)$ & $96(2.6 \%)$ & $102(2.8 \%)$ \\
\hline Missing BUSCOs & $160(4.4 \%)$ & $178(4.9 \%)$ & $349(9.6 \%)$ & $373(10.2 \%)$ \\
\hline Total BUSCO groups searched & 3640 & 3640 & 3640 & 3640 \\
\hline
\end{tabular}

Mbp) of the total assembly length was scaffolded into 19 chromosome-length scaffolds (Figure 3A). The number of chromosome-length scaffolds is consistent with the haploid number of chromosomes derived from karyotypes of females of two Callionymidae species (C. beniteguri and Repomucenus ornatipinnis) [23]. Therefore, the number of chromosomes appears to be relatively conserved within Callionymidae and it is likely that $C$. lyra follows the same chromosomal sex determination system as $C$. beniteguri and $R$. ornatipinnis ( $\$:$ $\left.\mathrm{X}_{1} \mathrm{X}_{2}-\mathrm{X}_{1} \mathrm{X}_{2}(2 n=38) ; \sigma: \mathrm{X}_{1} \mathrm{X}_{2}-\mathrm{Y}(2 n=37)\right)$ [23]. For a final assembly quality control, we mapped the raw nanopore reads with minimap2 v.2.17-r941 [24] and the DNBSeq data with bwa-mem v.0.7.17-r1194-dirty (RRID:SCR_010910) [25] onto the final assembly with a high mapping rate of $94.8 \%$ and $98.62 \%$, respectively. We further checked the assembly for contamination with BlobTools v.1.1.1 (RRID:SCR_017618) [26]. This analysis identified minor contamination from Proteobacteria (26 short contigs, in total $0.25 \mathrm{Mbp}$ ) and Uroviricota (2 contigs, in total $0.12 \mathrm{Mbp}$ ) (Figure 4). No contamination was found in the 19 chromosome-length scaffolds. Subsequently, we removed all contaminations and contigs with a length of $<200 \mathrm{bp}$ from the final assembly (for final statistics see Table 3 ). In addition, we screened for mitochondrial sequence contamination with BLASTN v.2.9.0+ (RRID:SCR_001598) [27] using the available mitochondrial genome sequence of $C$. lyra 

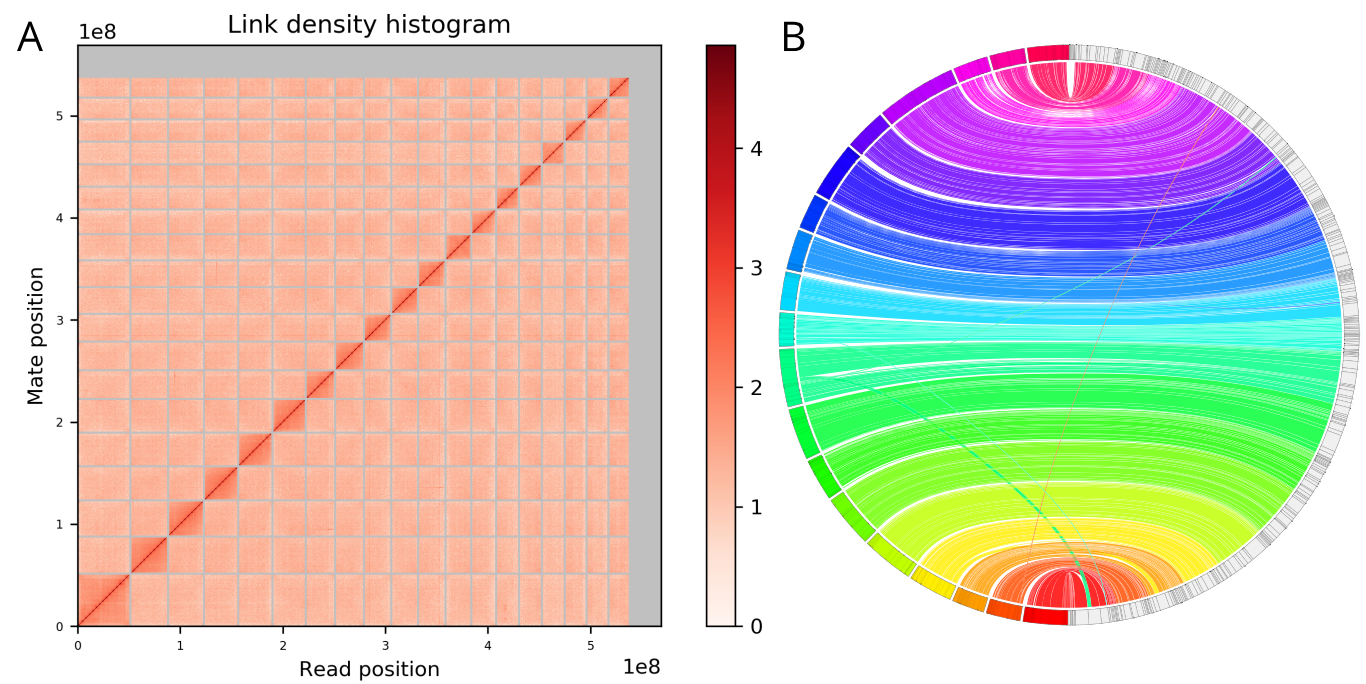

Figure 3. (A) Hi-C contact map of the 19 chromosome-length scaffolds, and additional unplaced scaffolds. (B) Whole genome synteny between the polished contig assembly from wtdbg2 (on the right) and the final Hi-C scaffolded chromosome-level assembly (on the left). Crossing lines indicate assembly artifacts corrected during scaffolding.

(Accession No.: MN122938.1) as a reference. A single sequence of mitochondrial origin (169 bp) was identified on one scaffold. This partial mitochondrial sequence could either be an assembly artifact or nuclear mitochondrial DNA (numt). A synteny plot comparing the polished wtdbg2 contig assembly with the final chromosome-level assembly, generated with JupiterPlot v.1.0 [28], found overall strong agreements with only few differences (Figure 3B). These likely constitute assembly errors in the contig assembly that were fixed by HiRise during scaffolding. A BUSCO analysis of the final assembly found slightly less complete BUSCO genes compared to the wdtbg2 contig assembly (94.5\% vs. 95.0\%) (Figure 2, Table 2).

\section{TRANSCRIPTOME ASSEMBLY AND QUALITY}

In addition to the genome, we assembled the transcriptome of $C$. lyra for subsequent use in the genome annotation using Trinity v.2.9.0 (RRID:SCR_013048) [29, 30] based on the $12.3 \mathrm{Gbp}$ multi-tissue RNAseq data. The resulting transcriptome assembly has a total length of 255.5 Mbp (Table 3). BUSCO analysis suggests a high transcriptome completeness with $87.8 \%$ of orthologous genes found in the transcriptome assembly (Figure 2, Table 2).

\section{GENOME ANNOTATION}

\section{Repeat annotation}

In order to annotate repeats in the assembly, we created a custom de novo repeat library using RepeatModeler v.1.0.11 (RRID:SCR_015027) [31] and combined this library with the Actinopterygii repeat database from RepBase. Repeats in the genome were then annotated using RepeatMasker open-4.0.7 (RRID:SCR_012954) [32]. Our analyses identified 27.66\% of repeats in the genome, of which the majority consisted of DNA transposons (6.10\%), LINE's (5.32\%) and simple repeats (3.47\%). Additionally, 10.69\% of unclassified repeats were identified (Table 4). 

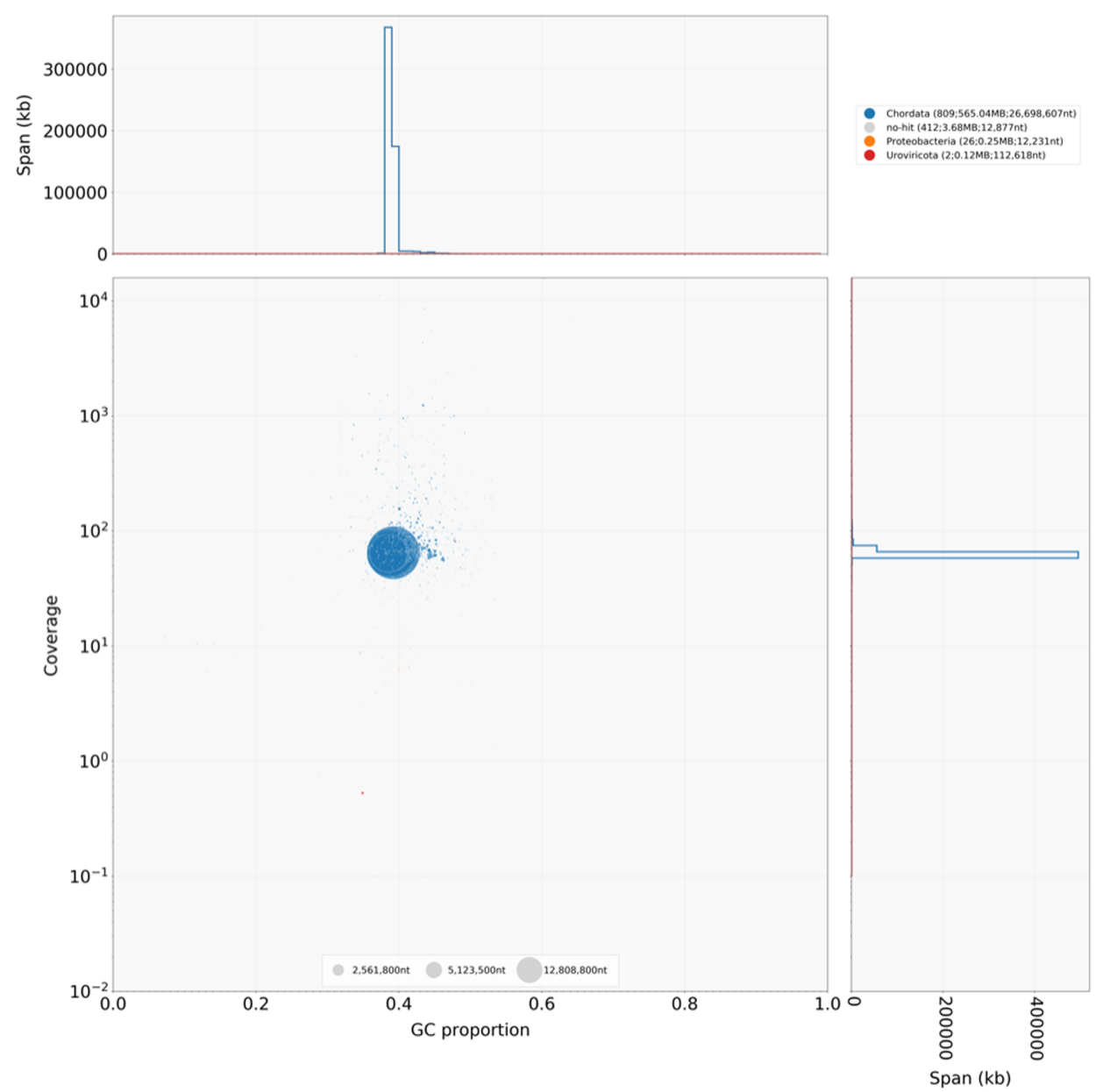

Figure 4. Blobtools plot showing the taxonomic assignments (blue colour for Chordata, gray for 'no hits', orange for Proteobacteria, and red for Uroviricota) of the different scaffolds, and scaffold-wide coverage and GC contents. The scaffolds were blasted against the NCBI nucleotide database. Scaffolds with assignments to Proteobacteria or Uroviricota were removed from the final assembly.

Table 3. Assembly statistics of the long-read based contig assembly (wtdbg2), Hi-C scaffolded assembly (HiRise) and the transcriptome assembly of Callionymus lyra.

\begin{tabular}{|cccc|}
\hline & wtdbg2 & HiRise $^{*}$ & Transcriptome \\
\hline No. of contigs & 1,782 & 1,205 & 246,012 \\
\hline No. of contigs (>1 kbp) & 1,707 & 1,151 & 66,332 \\
\hline L50 & 70 & 9 & 23,587 \\
L75 & 176 & 14 & 49,697 \\
\hline N50 & $2,201,294 \mathrm{bp}$ & $26,698,546 \mathrm{bp}$ & $2,787 \mathrm{bp}$ \\
\hline N75 & $856,699 \mathrm{bp}$ & $22,283,913 \mathrm{bp}$ & $1,474 \mathrm{bp}$ \\
\hline Max. contig length & $10,738,616 \mathrm{bp}$ & $51,234,906 \mathrm{bp}$ & $31,800 \mathrm{bp}$ \\
\hline Total length & $569,037,589 \mathrm{bp}$ & $568,707,486 \mathrm{bp}$ & $255,540,591 \mathrm{bp}$ \\
\hline GC (\%) & 38.97 & 38.97 & 46.84 \\
\hline No. of gaps & 0 & 57,348 & 0 \\
\hline No. of N’s per 100 kbp & 0.0 & 10.08 & 0.0 \\
\hline
\end{tabular}

*Final assembly after removing contaminated scaffolds and scaffolds $<200 \mathrm{bp}$. 


\begin{tabular}{|c|c|c|c|}
\hline Type of element & Number of elements & Length & Percentage of assembly \\
\hline SINES & 11,019 & $1,338,491$ & $0.24 \%$ \\
\hline LINEs & 117,005 & $30,245,708$ & $5.32 \%$ \\
\hline LTR elements & 21,355 & $6,054,779$ & $1.06 \%$ \\
\hline DNA transposons & 196,173 & $34,690,450$ & $6.10 \%$ \\
\hline Unclassified & 346,500 & $60,808,984$ & $10.69 \%$ \\
\hline Small RNA & 1,793 & 208,482 & $0.04 \%$ \\
\hline Satellites & 2,019 & 892,831 & $0.16 \%$ \\
\hline Simple repeats & 235,150 & $19,740,698$ & $3.47 \%$ \\
\hline \multirow[t]{2}{*}{ Low complexity } & 28,668 & $1,821,423$ & $0.32 \%$ \\
\hline & & Total: & $27.66 \%$ \\
\hline
\end{tabular}

\section{Gene annotation}

Prior to annotating genes, interspersed repeats in the genome were hard-masked and simple repeats soft-masked to increase the accuracy and efficiency of locating genes. Gene annotation was performed using MAKER2 v.2.31.10 (RRID:SCR_005309) [33]. First, evidence-based annotation was conducted using a combination of de novo assembled transcriptomes and homologous gene identification based on previously published proteins of the Tiger Tail Seahorse (Hippocampus comes) [3] and the SwissProt protein database [34]. Next, genes were $a b$ initio predicted with SNAP v.2006-07-28 (RRID:SCR_002127) [35] and Augustus v.3.3 (RRID:SCR_008417) [36]. The final gene annotation resulted in 19,849 transcripts, which is slightly lower compared to the number of transcripts in the Gulf Pipefish genome $(20,841)$ and Tiger Tail Seahorse genome $(22,941)$ [3, 4]. Of all identified gene models, 96\% had an AED score of $\leq 0.5$ (AED score distributions in GigaDB [14]), indicating a high quality of the annotated gene models [37]. In addition, BUSCO analysis identified $87.0 \%$ complete BUSCOs, which suggest a high completeness of the annotation (Figure 2, Table 2).

\section{CONCLUSION}

Here we report the first genome assembly of the 'benthic associated' Syngnathiformes clade, the sister group to the 'long-snouted clade' (e.g., seahorses and pipefish). The annotated genome of Callionymus lyra, with its high continuity (chromosome-level), provides an essential reference to study speciation and potential hybridization in Callionymidae and is an important resource for phylogenomic analyses among syngnathiform fish.

\section{DATA AVAILABILITY}

All raw data generated in this study including Nanopore long-reads, DNBSeq short-reads, Hi-C reads, and RNASeq data, and the chromosome-level assembly are accessible at GenBank under BioProject PRJNA634838. Annotation, results files and other data are available in the GigaDB repository [14].

\section{AUTHOR CONTRIBUTIONS}

SW, SP, MAN, MS, and AJ designed the study. SW, MN, AH, MK, RP, JT, JB, AK, SG, YS, CZ, KZ, DP, MM, and MMS performed laboratory procedures and sequencing. SP, JDR, RC, MW, MAN, MN, AH, MK, RP, JT, JB, AK, SG, YS, CZ, KZ, DP, MM, and SW conducted bioinformatic processing and analyses. All authors contributed to writing this manuscript. 


\section{LIST OF ABBREVIATIONS}

BLASTN: Basic Local Alignment Search Tool (for nucleotides); bp: base pairs; BUSCO: Benchmarking Universal Single-Copy Orthologs; DNBSeq: DNA NanoBall sequencing; Gbp: Gigabase pairs; hmwDNA: high molecular weight DNA; kbp: kilobase pairs; Mbp: megabase pairs; numt: nuclear mitochondrial DNA; ONT: Oxford Nanopore Technologies; pg: picogram; PI: propidium iodide; RNAseq: RNA sequencing.

\section{CONFLICT OF INTEREST}

The authors declare that they have no competing interests.

\section{CONSENT FOR PUBLICATION}

Not Applicable.

\section{ACKNOWLEDGEMENTS}

We thank Damian Baranski from the TBG laboratory centre for laboratory support. The present study is a result of the Centre for Translational Biodiversity Genomics (LOEWE-TBG) and was supported through the programme 'LOEWE - Landes-Offensive zur Entwicklung Wissenschaftlich-ökonomischer Exzellenz’ of Hesse’s Ministry of Higher Education, Research, and the Arts.

\section{REFERENCES}

1 Betancur RR, Wiley EO, Arratia G, Acero A, Bailly N, Miya M et al. Phylogenetic classification of bony fishes. BMC Evol. Biol., 2017; doi:10.1186/s12862-017-0958-3.

2 Longo SJ, Faircloth BC, Meyer A, Westneat MW, Alfaro ME, Wainwright PC, Phylogenomic analysis of a rapid radiation of misfit fishes (Syngnathiformes) using ultraconserved elements. Mol. Phylogenet. Evol., 2017; doi:10.1016/j.ympev.2017.05.002.

3 Lin Q, Fan S, Zhang Y, Xu M, Zhang H, Yang Y et al. The seahorse genome and the evolution of its specialized morphology. Nature, 2016; Nature Publishing Group; doi:10.1038/nature20595.

4 Small CM, Bassham S, Catchen J, Amores A, Fuiten AM, Brown RS et al. The genome of the Gulf pipefish enables understanding of evolutionary innovations. Genome Biol., 2016; doi:10.1186/s13059-016-1126-6.

5 Roth O, Solbakken MH, Tørresen OK, Bayer T, Matschiner M, Baalsrud HT et al. Evolution of male pregnancy associated with remodeling of canonical vertebrate immunity in seahorses and pipefishes. Proc. Natl Acad. Sci. USA, 2020; doi:10.1073/pnas.1916251117.

6 Froese R, Pauly D, FishBase. World Wide Web Electron. Publ. Version 122019. 2019; www.fishbase.org.

7 Fricke R, Annotated checklist of the dragonet families Callionymidae and Draconettidae (Teleostei: Callionymoidei), with comments on callionymid fish classification. Stuttg. Beitr. Naturk Ser. Biol., 645: 1-1032002.

8 Armstrong MJ, The predator-prey relationships of Irish Sea poor-cod (Trisopterus minutus L.), pouting (Trisopterus luscus L.) and cod (Gadus morhua L.). ICES J. Mar. Sci., 1982; Oxford Academic; doi:10.1093/icesjms/40.2.135.

9 Prost S, Winter S, De Raad J, Coimbra RTF, Wolf M, Nilsson MA et al. Education in the genomics era: Generating high-quality genome assemblies in university courses. GigaScience, 2020; doi:10.1093/gigascience/giaa058.

10 Mayjonade B, Gouzy J, Donnadieu C, Pouilly N, Marande W, Callot C et al. Extraction of high-molecular-weight genomic DNA for long-read sequencing of single molecules. BioTechniques, 2016; doi:10.2144/000114460.

11 Huang J, Liang X, Xuan Y, Geng C, Li Y, Lu H et al. BGISEQ-500 (DNBSEQ-G50) Sequencing. 2020; doi:10.17504/protocols.io.bimzkc76.

12 Marçais G, Kingsford C, A fast, lock-free approach for efficient parallel counting of occurrences of k-mers. Bioinformatics, 2011; doi:10.1093/bioinformatics/btr011. 
13 Vurture GW, Sedlazeck FJ, Nattestad M, Underwood CJ, Fang H, Gurtowski J et al. GenomeScope: fast reference-free genome profiling from short reads. Bioinformatics, 2017; doi:10.1093/bioinformatics/btx153.

14 Winter S, Prost S, De Raad J, Coimbra RTF, Wolf M, Marcel N et al. Supporting data for "Chromosome-level genome assembly of a benthic associated Syngnathiformes species: the common dragonet, Callionymus lyra.” GigaScience Database; http://dx.doi.org/10.5524/100799.

15 De Coster W, DHert S, Schultz DT, Cruts M, Van Broeckhoven C, NanoPack: visualizing and processing long-read sequencing data. Bioinformatics, 2018; doi:10.1093/bioinformatics/bty149.

16 Ruan J, Li H, Fast and accurate long-read assembly with wtdbg2. Nat Methods, 2020; doi:10.1038/s41592-019-0669-3.

17 Vaser R, Sović I, Nagarajan N, Šikić M, Fast and accurate de novo genome assembly from long uncorrected reads. Genome Res., 2017; doi:10.1101/gr.214270.116.

18 medaka. Oxford Nanopore Technologies; https://github.com/nanoporetech/medaka.

19 Walker BJ, Abeel T, Shea T, Priest M, Abouelliel A, Sakthikumar S et al. Pilon: An Integrated Tool for Comprehensive Microbial Variant Detection and Genome Assembly Improvement. PLOS One, 2014; doi:10.1371/journal.pone.0112963.

20 Gurevich A, Saveliev V, Vyahhi N, Tesler G, QUAST: quality assessment tool for genome assemblies. Bioinformatics, 2013; doi:10.1093/bioinformatics/btt086.

21 Seppey M, Manni M, Zdobnov EM, BUSCO: Assessing genome assembly and annotation completeness. Methods Mol Biol Clifton NJ, 2019; doi:10.1007/978-1-4939-9173-0_14.

22 Putnam NH, O’Connell BL, Stites JC, Rice BJ, Blanchette M, Calef R et al. Chromosome-scale shotgun assembly using an in vitro method for long-range linkage. Genome Res., 2016; doi:10.1101/gr.193474.115.

23 Murofushi M, Nishikawa S, Yosida TH, Cytogenetical Studies on Fishes. V. Proc. Jpn. Acad. Ser. B, 1983; doi:10.2183/pjab.59.58.

24 Li H, Minimap2: pairwise alignment for nucleotide sequences. Bioinformatics, 2018; doi:10.1093/bioinformatics/bty191.

25 Li H, Aligning sequence reads, clone sequences and assembly contigs with BWA-MEM 2013; arXiv preprint arXiv:13033997.

26 Laetsch DR, Blaxter ML, BlobTools: Interrogation of genome assemblies. F1000Research, 2017; doi:10.12688/f1000research.12232.1.

27 Zhang Z, Schwartz S, Wagner L, Miller W, A Greedy Algorithm for Aligning DNA Sequences. J. Comput. Biol., 2000; Mary Ann Liebert, Inc., publishers; doi:10.1089/10665270050081478.

28 Chu J, (2018, May 4), Jupiter Plot: A Circos-based tool to visualize genome assembly consistency (Version 1.0). Zenodo. http://doi.org/10.5281/zenodo.1241235.

29 Grabherr MG, Haas BJ, Yassour M, Levin JZ, Thompson DA, Amit I et al. Full-length transcriptome assembly from RNA-Seq data without a reference genome. Nat. Biotechnol., 2011; doi:10.1038/nbt.1883.

30 Haas BJ, Papanicolaou A, Yassour M, Grabherr M, Blood PD, Bowden J et al. De novo transcript sequence reconstruction from RNA-seq using the Trinity platform for reference generation and analysis. Nat. Protoc., 2013; doi:10.1038/nprot.2013.084.

31 RepeatModeler. http://www.repeatmasker.org/RepeatModeler/.

32 RepeatMasker. http://www.repeatmasker.org/RMDownload.html.

33 Holt C, Yandell M, MAKER2: an annotation pipeline and genome-database management tool for second-generation genome projects. BMC Bioinformatics, 2011; doi:10.1186/1471-2105-12-491.

34 The UniProt Consortium. UniProt: a worldwide hub of protein knowledge. Nucl. Acids Res., 2019; Oxford Academic; doi:10.1093/nar/gky1049.

35 Korf I, Gene finding in novel genomes. BMC Bioinformatics, 2004; doi:10.1186/1471-2105-5-59.

36 Stanke M, Diekhans M, Baertsch R, Haussler D, Using native and syntenically mapped cDNA alignments to improve de novo gene finding. Bioinformatics, 2008; doi:10.1093/bioinformatics/btn013.

37 Yandell M, Ence D, A beginner’s guide to eukaryotic genome annotation. Nat. Rev. Genet., 2012; Nature Publishing Group; doi:10.1038/nrg3174. 